DOI: $10.4274 /$ jarem.galenos.2021.3897

J Acad Res Med 2021;11(1):51-55

\title{
Vitamin D Deficiency is Associated with Depression, Anxiety and Sleep Disturbance in Pregnant Women
}

\author{
(1) Gonca Sağlam1, (1) Gizem Pektaş2, (1) Serdar Karakullukçu3, (1) Aras Pektaş2, (1) Demet Sağlam Aykut4 \\ ${ }^{1}$ Karadeniz Technical University Faculty of Medicine, Department of Physical Medicine and Rehabilitation, Trabzon, Turkey \\ ${ }^{2}$ Kaş State Hospital, Clinic of Obstetrics and Gynecology, Antalya, Turkey \\ ${ }^{3}$ Bayburt Community Health Center, Bayburt, Turkey \\ ${ }^{4}$ Karadeniz Technical University Faculty of Medicine, Department of Psychiatry, Trabzon, Turkey
}

Cite this article as: Sağlam G, Pektaş G, Karakullukçu S, Pektaş A, Sağlam Aykut D. Vitamin D Deficiency is Associated with Depression, Anxiety and Sleep Disturbance in Pregnant Women. J Acad Res Med 2021;11(1):51-55

\begin{abstract}
Objective: Vitamin D deficiency is prevalent in pregnancy and has been associated with psychological symptoms in non-pregnant patient populations. This study aimed to evaluate the relationship between depression, anxiety, sleep quality and vitamin D deficiency in pregnant women.

Methods: In this prospective cross-sectional study, data from a total of 153 pregnant women including demographic, pregnancy and laboratory were obtained. Participants were divided into two groups with low $(<20 \mathrm{ng} / \mathrm{mL})$ and normal $(\geq 20 \mathrm{ng} / \mathrm{mL})$ serum $25(\mathrm{OH})$ vitamin D levels. Pregnant women were screened for symptoms of depression and anxiety using the Beck depression inventory (BDI) and Beck anxiety inventory (BAI). Pittsburgh sleep quality index (PSOI) was utilised to investigate sleep quality. Groups were compared statistically.

Results: BDI, BAI and PSQI scores were significantly higher in patients with low vitamin D levels. Overall, 22.2\% of pregnant women had depression symptoms and $37.8 \%$ had moderate-severe anxiety symptoms in vitamin D deficient group.

Conclusion: Depression, anxiety and poor quality sleep during pregnancy were associated to vitamin D deficiency. This study emphasises the importance of screening for vitamin D deficiency among pregnant women.
\end{abstract}

Keywords: Vitamin D, pregnancy, depression, anxiety, sleep quality

\section{INTRODUCTION}

The high prevalence of depression and anxiety in pregnancy has been attributed to pregnancy-related anatomic, hormonal and physiologic changes. Women are at high risk for depression during pregnancy $(1,2)$. 10-15\% of women report depressive symptoms, whereas $4-7 \%$ are diagnosed with major depression during pregnancy $(2,3)$.
Many essential nutrients are required for a healthy nervous system and mood regulation $(4,5)$. Vitamin $D$ is one of those nutritional factors that may have neuroregulatory activity and act as a neuroactive hormone (6). This unique neurosteroid hormone may be involved in the anterior pituitary lobe functions, and its receptors are abundant across the human brain (7). Additionally, cytokines and inflammatory markers are enhanced by deficiencies of vitamin D via elevated concentrations of neuronal calcium. Literature

ORCID IDs of the authors: G.S. 0000-0001-7713-4435; G.P. 0000-0002-7454-4647; S.K. 0000-0003-1482-1312; A.P. 0000-0003-3968-1328; D.S.A. 0000-0002-8432-3290.

Corresponding Author/Sorumlu Yazar: Gonca Sağlam, E-mail: goncasaglam@hotmail.com
Received Date/Geliş Tarihi: 28.10.2020 Accepted Date/Kabul Tarihi: 22.01.2021

CC Copyright 2021 by University of Health Sciences Turkey, Gaziosmanpaşa Training and Research Hospital. Available on-line at www.jarem.org 
suggests the role of vitamin $D$ in neuro-immunomodulation and neuro-plasticity with various mechanisms (hypothalamic-pituitaryadrenal axis, insulin or serotonin mediated pathways, etc.) (8-10). It has been postulated that its deficiency alters neuro-transmitters such as serotonin, dopamine and noradrenaline, which are implicated to cause depressive symptoms (11). High prevalence of Vitamin D deficiency during pregnancy makes it a significant clinical problem.

Emerging lines of evidence suggest that vitamin $D$ also regulates immune system and decreases the release of inflammatory substances, including those that regulate sleep such as tumour necrosis factor-alpha and interleukin 1 (12). Vitamin D receptors that expressed in many brain areas may influence the daily sleepwake cycle. Association between inadequate vitamin D and sleep disorders is not well-understood; however, some observational researches suggest the connection of vitamin $D$ deficiency and poor sleep quality among non-pregnant populations $(13,14)$. Similarly, the protective effect of vitamin D on mood disorders, mostly depression, in non-pregnant people has been reported previously in literature. However, results have not been entirely consistent due to the complexity of this connection. In addition, this research area was rarely conducted among pregnant women, and the relationship of vitamin $D$ and anxiety symptoms is underinvestigated in pregnancy. In this study, we investigated the vitamin $D$ effect on depression and anxiety symptoms and examine the frequency of sleep disturbances with the goal of clarifying the association between inadequate vitamin $D$ and sleep disorders risk in pregnant women.

\section{METHODS}

This study was designed as a prospective cross-sectional study. The study population for this report is from the first 200 pregnant women who attended an outpatient obstetric clinic for routine exam for pregnancy in each trimester. Pregnant women were interviewed in the period of April 2018 and May 2018. Inclusion criteria includes the following: 18 years or older, no schizophrenia or other psychiatric history, restless legs syndrome, drug addiction, psychiatric drug use, any chronic musculoskeletal disorder leading to poor sleep quality, sleep disturbance history before pregnancy and comorbidities such as diabetes and hypertension. Consequently, 153 pregnant women were available for the analysis.

Pregnant women were screened for depression symptoms using the Beck depression inventory (BDI) and Beck anxiety inventory (BAl) for anxiety symptoms $(15,16)$.

$\mathrm{BDI}$ is a 21-item self-report scale used to assess the current severity of depression symptoms. Each item is rated in a four-point scale (0-3) with possible total scores ranging from 0 to 63. A cutting score of 17 or over represents depression. Turkish validity and reliability analysis of this scale was reported by Hisli (17). The BAI consists of 21 self-reported items (four-point scale) to assess the intensity of physical and cognitive anxiety symptoms during the past week. Scores may range from 0 to 63 evaluated as follows:
Mild anxiety (8-15), moderate anxiety (16-25) and severe anxiety (26-63). The Turkish version of this inventory was devised by Ulusoy (18). The Pittsburgh sleep quality index (PSOl) was used to assess sleep quality over the previous month. In this scale, a total sum of $\geq 5$ indicates low sleep quality (19). The PSQI was demonstrated to have good validity and reliability among Turkish population (20).

The immune chemiluminometric assay (Architect i2000, Abbott, Germany) was used to determine vitamin $D$ levels in patients. It has been accepted that serum 25-hydroxyl vitamin D [25 (OH) D] of $<20 \mathrm{ng} / \mathrm{mL}$ is the level of vitamin D deficiency (21). Participants were divided into two groups according to serum $25(\mathrm{OH}) \mathrm{D}$ levels: 90 patients with serum $25(\mathrm{OH})$ D levels of $<20 \mathrm{ng} / \mathrm{mL}$ comprising the vitamin D deficient group (group 1) and 63 women with serum $25(\mathrm{OH}) \mathrm{D}$ levels of $\geq 20 \mathrm{ng} / \mathrm{mL}$ comprising the vitamin D sufficient group (group 2).

This study was approved and initiated by the Clinical Research Ethics Committee of Karadeniz Technical University Faculty of Medicine (date: 02.10.2017, protocol no: 142). All participants were informed about the research concept, and written informed consent was obtained from all participants.

\section{Statistical Analysis}

Data were analysed using Statistical Package for the Social Sciences 23.0 statistics programme. Chi-square test was used for categorical variables. Descriptive statistics of evaluation results were as follows: Number and percentage for categorical variables, mean and standard deviation for numerical variables. The normality of variables was assessed using KolmogorovSmirnov test. Mann-Whitney $U$ test was used for comparison of groups, and correlation analysis was done by Spearman test. Interpreting the correlation coefficients was based on following points: Values between 0.3 and 0.5 indicate a low positive linear relationship, values between 0.5 and 0.7 indicate a moderate positive linear relationship, values between 0.7 and 0.9 indicate a high positive linear relationship and values between 0.9 and 1 indicate a very high positive linear relationship (22). Cronbach's alpha values were evaluated as follows: $0.5 \leq \alpha<0.6$ indicating a poor internal consistency, $0.6 \leq \alpha<0.7$ indicating a fair internal consistency, $0.7 \leq \alpha<0.9$ indicating a good internal consistency and $\geq 0.9$ indicating an excellent/strong internal consistency (23). A probability level of $<0.05$ was considered as statistically significant.

\section{RESULTS}

\section{Socio-demographic and Laboratory Parameters}

Data from a total of 90 participants in group 1 and 63 participants in group 2 were reviewed. No significant differences were determined for age, number of previous pregnancies, gestational week, pre-pregnancy smoking history and concomitant diseases of pregnant women in both groups. Mean values for serum haemoglobin, ferritin, thyroxin, thyroid-stimulating hormone and calcium in both groups were similar. The socio-demographic and laboratory parameters are summarised in Table 1. 


\section{Evaluation of Depression and Anxiety}

Among all participants with vitamin D deficiency (group 1), mean score of BDI was $14.8 \pm 10.3$, whereas mean BAl score was $15.3 \pm 8.7$. $\mathrm{BDI}$ and BAl scores were significantly higher in group $1(p<0.001)$ (Table 1). In group 1, $22.2 \%(n=20)$ had high $B D I$ values, whereas $0 \%$ in group 2 . In group 1, 6.2\% had mild, $25.6 \%$ had moderate and $12.2 \%$ had severe anxiety symptoms. In group $2,96.8 \%$ had mild and $3.2 \%$ had moderate level of anxiety symptoms $(p<0.001)$ (Table 2).

\section{Sleep Quality}

According to PSOI screening, the mean score was $9.4 \pm 5.6$ in group 1 indicating low sleep quality, whereas $2.6 \pm 3.1$ in group 2. PSOI points were significantly worse in the vitamin $D$ deficient

\begin{tabular}{|c|c|c|c|}
\hline & $\begin{array}{l}\text { Group } 1(n=90) \\
\text { mean } \pm S D\end{array}$ & $\begin{array}{l}\text { Group } 2(n=63) \\
\text { mean } \pm S D\end{array}$ & $p$ \\
\hline Age (year) & $27.4 \pm 5.7$ & $27.9 \pm 5.9$ & 0.467 \\
\hline $\begin{array}{l}\text { Number of previous } \\
\text { pregnancies }\end{array}$ & $1.8 \pm 1.0$ & $1.6 \pm 0.8$ & 0.145 \\
\hline Gestational week & $27.6 \pm 10.8$ & $28.1 \pm 6.9$ & 0.340 \\
\hline $\begin{array}{l}\text { Pre-pregnancy } \\
\text { smoking history }\end{array}$ & $7.7 \%(n=7)$ & $9.5 \%(n=6)$ & 0.931 \\
\hline Haemoglobin (g/dL) & $11.5 \pm 1.4$ & $11.7 \pm 1.1$ & 0.544 \\
\hline Ferritin (ng/mL) & $26.0 \pm 7.6$ & $25.2 \pm 10.0$ & 0.359 \\
\hline T4 & $1.2 \pm 0.5$ & $1.2 \pm 0.3$ & 0.753 \\
\hline TSH & $2.3 \pm 2.1$ & $2.2 \pm 1.4$ & 0.838 \\
\hline Calcium (mg/dL) & $8.9 \pm 0.5$ & $8.9 \pm 0.7$ & 0.780 \\
\hline BDI & $14.8 \pm 10.3$ & $3.6 \pm 3.0$ & $<0.001$ \\
\hline BAl & $15.3 \pm 8.7$ & $4.1 \pm 3.8$ & $<0.001$ \\
\hline PSQl & $9.4 \pm 5.6$ & $2.6 \pm 3.1$ & $<0.001$ \\
\hline
\end{tabular}

BAI: Beck anxiety inventory, BDI: Beck depression inventory, SD: standard deviation, PSOI: Pittsburgh sleep quality index, T4: thyroxine hormone, TSH: thyroid-stimulating hormone

Table 2. Comparison of BDI, BAI and PSOI between groups

\begin{tabular}{|l|l|l|l|l|}
\hline & $\begin{array}{l}\text { Group 1, } \\
(\%)\end{array}$ & $\begin{array}{l}\text { Group 2, } \\
\mathbf{n}(\%)\end{array}$ & $\mathbf{p}$ & $\begin{array}{l}\text { Total, } \\
\mathbf{n}(\%)\end{array}$ \\
\hline BDI: $0-17$ & $70(77.8)$ & $63(100.0)$ & $<0.001$ & $133(86.9)$ \\
\hline BDI $\geq 17$ & $20(22.2)$ & $0(0.0)$ & $<0.001$ & $20(13.1)$ \\
\hline BAl: $8-15$ & $56(6.2)$ & $61(96.8)$ & $<0.001$ & $117(76.5)$ \\
\hline BAI: $16-25$ & $23(25.6)$ & $2(3.2)$ & $<0.001$ & $25(16.3)$ \\
\hline BAI $\geq 26$ & $11(12.2)$ & $0(0.0)$ & $<0.001$ & $11(7.2)$ \\
\hline PSQI: $0-4$ & $13(14.4)$ & $55(87.3)$ & $<0.001$ & $68(44.4)$ \\
\hline PSQI $\geq 5$ & $77(85.6)$ & $8(12.7)$ & $<0.001$ & $85(55.6)$ \\
\hline
\end{tabular}

BAI: Beck anxiety inventory, BDI: Beck depression inventory, PSOI: Pitt burgh sleep quality index group compared to that of the vitamin D sufficient group $(p<0.001)$ (Table 2). In group 1, 85.6\% had sleep impairment, whereas $12.7 \%$ in group 2.

A high positive correlation was found between $\mathrm{BDI}$ and PSQI scores of pregnant women $(r=0.755) \quad(p<0.001)$. Correlation between BAI and PSOI scores of pregnant women was also examined, and a statistically significant high positive correlation was found (Table 3). The Cronbach's alpha values for scales used in this study indicated a strong internal consistency $(0.945$ for BDI, 0.913 for BAl and 0.951 for PSQl).

Table 3. The correlations between PSOI, BDI and BAI scores

\begin{tabular}{l|l|l|} 
& BAI & BDI \\
\hline PSOI & $r=0.724$ & $r=0.755$ \\
& $p<0.001$ & $p<0.001$
\end{tabular}

BAI: Beck anxiety inventory, BDI: Beck depression inventory, PSOI: Pitt burgh sleep quality index

\section{DISCUSSION}

Depression and anxiety were reported as the most common psychiatric disorders that affect up to $15 \%$ of pregnant women and are the leading causes of disease-related disability among women. Several studies have investigated the evidence for a causal link between low vitamin D levels and mood disorders in pregnancy. Results remain controversial; however, vitamin D deficiency has been associated with an increased risk of depression in some studies (24-27). In this study, the incidence of depression, anxiety and sleep disturbance were found to be higher in vitamin $\mathrm{D}$ deficient pregnant women compared to that of the vitamin $\mathrm{D}$ sufficient pregnant women. Limited data was found regarding the relationship between low vitamin $D$ levels and presence of anxiety (28), thus, our study revealed that vitamin D deficiency is connected with elevated anxiety symptoms as well as depressive symptoms. This result proves the fact that insufficient level of vitamin $D$ in pregnant women is linked to psychosocial stress and mood disorders.

A systematic review examined protective function of vitamin $\mathrm{D}$ on perinatal depression, and authors indicated a significant association between inadequate vitamin $D$ and depression in pregnancy. A total of ten studies on vitamin D were included, eight were prospective cohort studies, and six of them reported vitamin $D$ deficiency as predictive for increased risk of depression during pregnancy. The remaining four studies could not show any significant relationship between vitamin $D$ levels and depression (29). Another study on this topic systematically reviewed literature, mostly observational studies, to show whether vitamin $D$ status might influence the possibility of perinatal depression. Significant associations were found between vitamin D deficiency and depressive symptoms among pregnant women in Caucasian, Chinese, Iranian and Turkish populations (30). A cross-sectional cohort investigated the association of vitamin $D$ levels and depression and anxiety symptoms in early pregnancy with 498 
women. Participants were screened by depression, anxiety, and stress scales (DASS-21) and patient health questionnaire depression module (PHO-9) instruments. This study suggested that low vitamin D status is related to self-reported symptoms of depression in early pregnancy. Results show that $12 \%$ of women had moderate anxiety and depression. A $1 \mathrm{ng} / \mathrm{mL}$ reduction in serum $25(\mathrm{OH})$ vitamin D concentrations was associated with 0.043 and 0.040 higher DASS-21 anxiety and PHQ-9 scores, respectively. Since vitamin $D$ deficiency was not prevalent in patients in this study, the mean $25(\mathrm{OH})$ vitamin D level for this study was 34.4 $\mathrm{ng} / \mathrm{mL}$ (28). Nielsen et al. (25) measured serum concentrations of $25(\mathrm{OH})$ vitamin $\mathrm{D}$ in 605 women with postpartum depression and 875 in control. In contrast to the above mentioned studies, this case-control study revealed that an increase in vitamin D levels enhances the prevalence of postnatal depression. This unexpected result was explained with genetic differences.

For the present study, tools for determining depression and anxiety symptoms were different from previous studies which mainly used Edinburgh postnatal depression scale (EPDS). We measured the mood disorder symptoms of pregnant women using the screening instruments of BDI and BAI, which are widely used scales, similar with EPDS, although not diagnostic; however, highly correlated with physicians diagnostic opinion. A cut-off point of 17 was used to identify depression using BDI, and $22.2 \%$ of vitamin $D$ deficient group was defined to have depressive symptoms. Williams et al. (26) observed patients with BDI according to the same vitamin $D$ threshold level with our study and found inverse association between vitamin $\mathrm{D}$ status and depression scores in early and late pregnancy but not postpartum. Mean BDI scores at 12-20 gestational weeks for vitamin $D$ deficient group $(n=98)$ and vitamin D sufficient group $(n=19)$ were 7.4.2 \pm 4.9 and 10.2 \pm 7.1 , respectively. In this present study, BAl scores of pregnant women were also investigated and classified in three sections. Anxiety symptoms were found to be associated with vitamin $D$ deficiency. Results demonstrated that; $6.2 \%$ of vitamin $D$ deficient group had mild, $25.6 \%$ had moderate and $12.2 \%$ had severe anxiety symptoms.

Sleep deprivation enhances the risk of gestational complications; however, few studies focused on sleep quality across pregnancy. An internet based survey demonstrated that $76 \%$ of women suffers from poor sleep quality across all months of pregnancy (31). In the current study, a significant relationship was observed between low vitamin $D$ status and sleep disturbances with the average gestational week of $27.6 \pm 10.8$ (group 1) and $28.1 \pm 6.9$ (group 2). Notably, the prevalence of unhealthy sleep among vitamin D deficient group was $85.6 \%$ as defined by PSQI score of $>5$. Similar to our findings, vitamin $D$ deficiency was found to be a risk factor of unhealthy sleep in a meta-analysis by Gao et al. (32) that reported vitamin D deficiency (cut-off value: $20 \mathrm{ng} /$ $\mathrm{mL}$ ) increases the incidence of sleep disorders by approximately $60 \%$. The underlying mechanism of this relationship is currently unknown; however, the possible hypothesis is that vitamin D plays a role in circadian rhythms (33). Furthermore, vitamin D receptors are common in human brain, such as the hypothalamus, prefrontal cortex, substantia nigra and raphe nuclei, which are known to execute important roles in sleep (34). Cheng et al. (35) and colleagues provided evidence for vitamin $D$ deficiency related to poor sleep quality at mid-pregnancy, which is consistent with our findings. They examined sleep quality by PSOI, which yielded scores of 7.04 \pm 3.47 for vitamin $D$ deficient group indicating poor sleep quality. Vitamin D deficient group in our study had mean PSQl of $9.4 \pm 5.6$ regarding sleep disturbances, whereas the control group had lower PSQI scores. Another related study was conducted among women in the last trimester of pregnancy. In contrast to our study, this study showed no statistical significant difference in PSQI scores between the vitamin D deficient group and vitamin D sufficient groups (36).

Strengths of our research include the following: A substantial study population of vitamin D deficient participants making comparable analyses possible, $58.8 \%$ of participants had severe vitamin D deficiency. Moreover, psychological status was assessed with well validated, internationally and widely used questionnaires. Study population was limited after eliminating participants with previous psychiatric diseases and chronic musculoskeletal disorders. In addition, an important limitation includes the fact that depression and anxiety were not systematically evaluated by psychiatrists. Furthermore, no power analysis was performed to determine the optimal sample size before the initiation of the study.

Further interventional researches should also address depression and anxiety given the impact of vitamin D on the psychological well-being in pregnancy. This is one of the few studies that have comprehensively evaluated the association of depression and anxiety and low vitamin D levels across pregnancy. Significant relationships were observed between $\mathrm{BDI}$ and $\mathrm{BAI}$ scores and vitamin $D$ deficiency. Results of this study provided additional evidence that a strong relationship between poor sleep quality and low vitamin D levels is present, whereas BAI and BDI scores were correlated with PSOI scores as expected. Previous studies have not examined psychological symptoms and sleep quality among pregnant women beside the fact that depression is linked with sleep disorders. Risk for mood disorders was determined to be higher in vitamin $\mathrm{D}$ deficient group and this may influence the association of vitamin D levels and sleep quality; however, this study suggest the relationship between inadequate vitamin $D$ and sleep disorders in pregnant women.

\section{CONCLUSION}

Vitamin $D$ deficiency can have a negative impact on pregnant woman as it may induce depressive and anxiety symptoms and worsen sleep quality. High-quality studies in deficient populations with large sample sizes should be carried out to verify this relationship and determine the effect of vitamin $D$ supplementation in sleep disorders among pregnant women and strengthen the evidence base on this topic.

Acknowledgements: Authors would like to thank the pregnant women who participated in this study. 
Ethics Committee Approval: This study was approved and initiated by the Clinical Research Ethics Committee of Karadeniz Technical University Faculty of Medicine (date: 02.10.2017, protocol no: 142).

Informed Consent: All participants were informed about the research concept, and written informed consent was obtained from all participants.

Peer-review: Externally peer-reviewed.

Author Contributions: Surgical and Medical Practices - G.P., A.P.; Concept - G.S.; Design - G.S.; Data Collection and/or Processing - G.S., G.P., S.K., Analysis and/or Interpretation - S.K.; Literature Search - G.S.,G.P.; Writing Manuscript - G.S., D.S.A.

Conflict of Interest: The authors have no conflict of interest to declare.

Financial Disclosure: The authors declared that this study has received no financial support.

\section{REFERENCES}

1. Ryan D, Milis L, Misri N. Depression during pregnancy. Can Fam Physician 2005; 51: 1087-93.

2. Patkar AA, Bilal L, Masand PS. Pharmacotherapy of depression in pregnancy. Ann Clin Psychiatry 2004; 16: 87-100.

3. Andersson L, Sundstrom-Poromaa I, Bixo M, Wulff M, Bondestam K, Astrom M. Point prevalence of psychiatric disorders during the second trimester of pregnancy: a population-based study. Am J Obstet Gynecol 2003; 189: 148-54.

4. Grote NK, Bridge JA, Gavin AR, Melville JL, lyengar S, Katon WJ. A metaanalysis of depression during pregnancy and the risk of preterm birth, low birth weight, and intrauterine growth restriction. Arch Gen Psychiatry 2010; 67: 1012-24.

5. Rechenberg K, Humphries D. Nutritional interventions in depression and perinatal depression. Yale J Biol Med 2013; 86: 127.

6. Bodnar LM, Wisner KL. Nutrition and depression: implications for improving mental health among childbearing-aged women. Biol Psychiatry 2005; 58: 679-85.

7. Berridge MJ. Vitamin D: A custodian of cell signalling stability in health and disease. Biochem Soc Trans 2015; 43:349-58.

8. Sarris J. Clinical use of nutraceuticals in the adjunctive treatment of depression in mood disorders. Australas Psychiatry 2017; 25: 369-72.

9. Berridge JM. Vitamin D and Depression: Cellular and Regulatory Mechanisms. Pharmacol 2017; 69: 80-92.

10. Anglin RE, Samaan Z, Walter SD, McDonald SD. Vitamin D deficiency and depression in adults: systematic review and meta-analysis. Br J Psych 2013; 202: 100-7.

11. Fernandez de Abreu DA, Eyles D, Féron F. Vitamin D, a neuroimmunomodulator: implications for neurodegenerative and autoimmune diseases. Psychoneuroendocrinology, 2009; 34(Suppl 1): S265-77.

12. Krueger JM, Majde JA, Rector DM. Cytokines in immune function and sleep regulation. Handbook Clin Neurol 2011; 98: 229.

13. Mccarty DE, Reddy A, Keigley $Q$, Kim PY, Marino AA. Vitamin D, race, and excessive daytime sleepiness. J Clin Sleep Med 2012; 6: 693-7.

14. McCarty DE, Reddy A. Prevalence of vitamin D insufficiency/deficiency among sleep medicine patients complaining of somatic pain and correlation with daytime sleepiness. Sleep 2011; (Supplement 35): A230.

15. Beck AT, Ward CH, Mendelson M. An inventory for measuring depression. Arch Gen Psychiatry 1961; 4: 561-71.

16. Beck AT, Epstein N, Brown G, Steer RA, Mock J, Erbaugh J. An inventory for measuring clinical anxiety: psychometric properties. J Consult Clin Psychol 1988; 56: 893-7.
17. Hisli N. A study on the validity of Beck Depression Inventory. Psychiatry Journal 1988; 6: 118-22.

18. Ulusoy M. Beck anxiety inventory: a validity and reliability study. Unpublished specialty thesis, Istanbul: Bakırköy Mental Health and Neurological Diseases. 1993.

19. Buysse DJ, Reynolds CF, Monk TH, Berman SR, Kupfer DJ. The Pittsburgh Sleep Quality Index: a new instrument for psychiatric practice and research. Psychiatry Res 1989; 28: 193-213.

20. Agargün MY, Kara $H$, Anlar $O$. The validity and reliability of the Pittsburgh Sleep Quality Index. Turk Psikiyatr Derg 1996; 7: 107-15.

21. Holick MF. High prevalence of vitamin D inadequacy and implications for health. Mayo Clin Proc 2006; 81: 353-73.

22. Mukaka MM. Statistics corner: A guide to appropriate use of correlation coefficient in medical research. Malawi Med J 2012; 24: 69-71.

23. Kilıç S. Cro.nbach's alpha reliability coefficient. Psychiatr Behav Sci 2016; 6: 47-8.

24. Shakeel N, Eberhard-Gran M, Sletner L, Slinning K, Martinsen EW, Holme I, et al. A prospective cohort study of depression in pregnancy, prevalence and risk factors in a multi-ethnic population. BMC Pregnancy Childbirth. 2015; 15 : 5.

25. Nielsen NO, Strom M, Boyd HA, Andersen EW, Wohlfahrt J, Lundqvist $M$ et al. Vitamin $D$ status during pregnancy and the risk of subsequent postpartum depression: a case-control study. PLoS One 2013; 8: e80686.

26. Williams JA, Romero VC, Clinton CM, Vazquiz DM, Marcus SM, Chilimigras $\mathrm{JL}$, et al. Vitamin $\mathrm{D}$ levels and perinatal depressive symptoms in women at risk: a secondary analysis of the mothers, omega-3, and mental health study. BMC Pregnancy Childbirth 2016; 16: 203.

27. Miyake Y, Tanaka K, Okubo H, Sasaki S, Arakawa M. Dietary vitamin D intake and prevalence of depressive symptoms during pregnancy in Japan. Nutrition. 2015; 31: 160-5.

28. Huang JY, Arnold D, Qiu CF, Miller RS, Williams MA, Enquobahrie DA. Association of serum vitamin $\mathrm{D}$ with symptoms of depression and anxiety in early pregnancy. J Women Health 2014; 23: 588-95.

29. Sparling TM, Nesbitt RC, Henschke N, Gabrysch S. Nutrients and perinatal depression: a systematic review. J Nutr Sci 2017; 6: e61.

30. Aghajafari F, Letourneau N, Mahinpey N, Cosic N, Giesbrecht G. Vitamin $\mathrm{D}$ deficiency and antenatal and postpartum depression: a systematic review. Nutrients 2018; 10: 478.

31. Mindell JA, Cook RA, Nikolovski J. Sleep patterns and sleep disturbances across pregnancy. Sleep Med 2015; 16: 483-8.

32. Gao $Q$, Kou T, Zhuang B, Ren Y, Dong X, Wang $Q$. The Association between vitamin $D$ deficiency and sleep disorders: a systematic review and meta-analysis. Nutrients 2018; 10: 1395.

33. Gutierrez-Monreal MA, Duran RCD, Moreno-Cuevas JE, Scott SP. A role for 1,25-dihydroxyvitamin D3 in the expression of circadian genes. J Biol Rhythms 2014; 29: 384-8.

34. Facco FL, Kramer J, Ho KH, Zee PC, Grobman WA. Sleep disturbances in pregnancy. Obstet Gynecol 2010; 115: 77-83.

35. Cheng T, Cheung Y, Cai S, Colega M, Godfrey K, Chong YS, et al. Plasma vitamin $d$ deficiency is associated with poor sleep quality and night-time eating at mid-pregnancy in Singapore. Nutrients 2017; 9: 340.

36. Gunduz S, Kosger H, Aldemir S, Akcal B, Tevrizci H, Hizli et al. Sleep deprivation in the last trimester of pregnancy and inadequate vitamin $D$ : Is there a relationship? J Chin Med Assoc 2016; 79: 34-8. 